\title{
Mucormicosis rino-órbito-cerebral crónica: una patología poco frecuente
}

\section{Chronic rhino-orbital-cerebral mucormycosis: an uncommon pathology}

\author{
Karen García C. ${ }^{1}$, Rafael Iracheta G. ${ }^{1}$, Álvaro Salas Z. ${ }^{2}$, \\ Neil Saldías V. ${ }^{3}$, Ricardo Zamorano S. ${ }^{1}$, Paul Boettiger B. ${ }^{1}$
}

\section{Resumen}

La mucormicosis rino-órbito-cerebral (ROC) crónica es una patología poco frecuente, con un número reducido de casos publicados en la literatura, cuyas manifestaciones son muy diversas e inespecíficas. El tratamiento se basa en la experiencia de casos y series de casos. Las herramientas terapéuticas incluyen el uso de antifúngicos endovenosos y orales por tiempo prolongado, asociado o no a debridamiento quirúrgico amplio, pudiendo requerir incluso exenteración orbitaria. Presentamos a continuación un caso de mucormicosis ROC crónica, junto con las dificultades para su diagnóstico y manejo, en el que destaca el enfrentamiento multidisciplinario. Dada la poca frecuencia de esta enfermedad, nos parece relevante difundirlo.

Palabras clave: mucormicosis, rino-órbito-cerebral, fúngico.

\begin{abstract}
Chronic rhino-orbital-cerebral mucormycosis is a rare condition with a small number of cases that have been published, whose manifestations are very diverse and nonspecific. The treatment is based on case series experiences. Therapeutic options include the use of long-term intravenous and oral antifungals, associated or not with extensive surgical debridement, and may even require orbital exenteration. We present below a case of chronic rhino-orbital-cerebral mucormycosis with the challenge of diagnosis and management in which multidisciplinary work is fundamental. Since it is an uncommon pathology, it seems relevant to share the information.
\end{abstract}

Keywords: mucormycosis, rhino-orbital-cerebral, fungal.

\author{
'Departamento de \\ Otorrinolaringología, Hospital \\ Clínico Universidad de Chile. \\ Santiago, Chile. \\ ${ }^{2}$ Departamento de Radiología, \\ Hospital Clínico Universidad \\ de Chile. Santiago, Chile. \\ ${ }^{3}$ Departamento de \\ Oftalmología, Hospital \\ Clínico Universidad de Chile. \\ Santiago, Chile. \\ Los autores declaran no tener \\ conflictos de interés. \\ Recibido el 25 de agosto de \\ 2019. Aceptado el 10 de abril \\ de 2020. \\ Correspondencia: \\ Paul Boettiger B. \\ Santos Dumontt 999, Hospital \\ Clínico Universidad de Chile. \\ Santiago, Chile. \\ Email: paulboettiger@gmail.com
}

\section{Introducción}

La mucormicosis es una infección oportunista severa que afecta predominantemente a pacientes inmunocomprometidos, sin embargo, existen reporte de casos en pacientes inmunocompetentes, que corresponden al $4 \%-43 \%{ }^{1-4}$. Si bien, la mucormicosis crónica puede afectar la piel, pulmón o riñón, la infección rino-órbito-cerebral (ROC) es la más frecuente $e^{5-7}$. En Chile la incidencia y prevalencia de infecciones fúngicas es desconocida. Se estima que las infecciones fúngicas pulmonares son las más frecuentes y la incidencia de mucormicosis en el país sería de 0,2/100.000 habitantes ${ }^{8}$. Según su presentación, puede ser clasificada en aguda y crónica. La mayoría de las mucormicosis invasivas se presentan en forma aguda, constituyendo la forma crónica un total de 23 casos publicados entre los años 1964 y $2014^{1}$. No existe una definición bien establecida para la presentación crónica (o indolente, según otros autores), con síntomas que varían de semanas a meses. Sin embargo, lo más aceptado, es un cuadro de más de 4 semanas de evolución, con un promedio de 7 meses en los casos publicados ${ }^{2}$. En esta revista, el año 2008, se publicó la experiencia de mucormicosis ROC, incluyendo 16 pacientes, de los cuales sólo dos se presentaron en forma crónica ${ }^{9}$. Presentamos a continuación, a nuestro conocimiento, el tercer caso clínico 
de mucormicosis crónica ROC reportado en Chile. Se discute la clínica, junto con las dificultades en su estudio y manejo, con la finalidad de divulgar entre los lectores la existencia de esta patología. Debido a que es una enfermedad muy infrecuente, la literatura se basa en publicación de casos clínicos, sin desarrollo de ensayos clínicos aleatorizados doble ciego ${ }^{1,2}$. Por esto, resulta fundamental compartir esta experiencia.

\section{Caso Clínico}

Paciente de sexo femenino, de 47 años, con antecedentes de diabetes mellitus tipo II con mal control metabólico e hipertensión arterial. Presenta historia de 2 meses de evolución con síntomas respiratorios altos. Evoluciona con dolor ocular izquierdo, asociado a ptosis palpebral y oftalmoplejía ipsilateral. Previo a consulta recibe tratamiento por odontología por pieza dentaria infectada con extracción y amoxicilina oral. Evaluada por oftalmología en

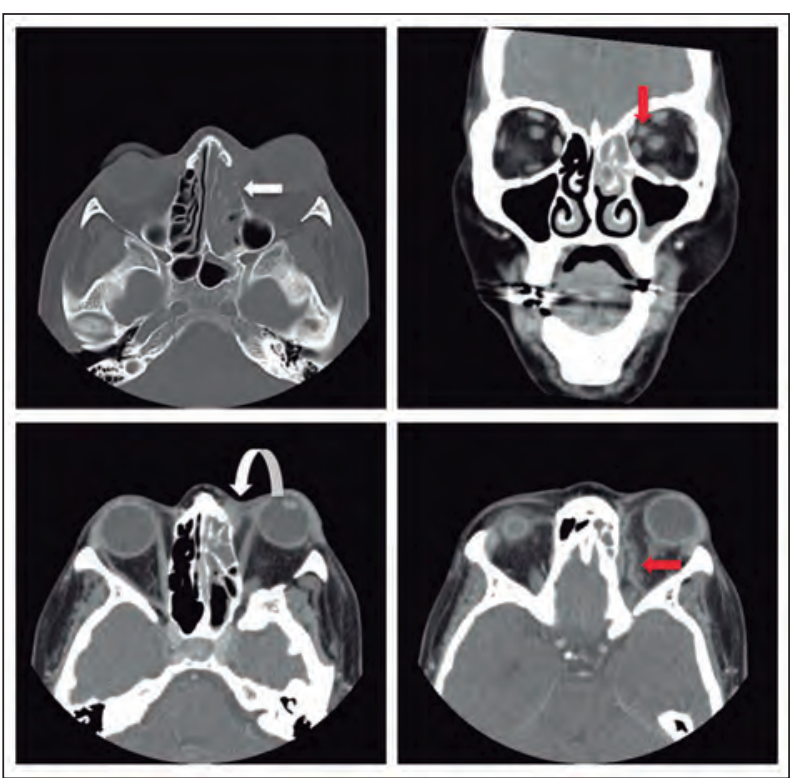

Figura 1. Tomografía computada de órbitas demuestra compromiso inflamatorio del laberinto etmoidal, osteólisis de lámina papirácea (flecha blanca), compromiso del saco lagrimal (flecha curva) y proptosis izquierda. Aumento de densidad de la grasa intraconal y dilatación de la vena oftálmica superior (flecha roja), sin evidente realce tras el uso de contraste endovenoso, sugerente de celulitis orbitaria y tromboflebitis. hospital de origen donde destaca edema palpebral con pupila arreactiva y proptosis izquierda, acompañado de dolor y limitación a la movilidad ocular. Es hospitalizada de urgencia con diagnóstico de celulitis orbitaria izquierda en contexto de diabetes mellitus descompensada. Recibe tratamiento con vancomicina durante 7 días, con respuesta clínica parcial. A los 7 días evoluciona con disminución de eritema, persistiendo proptosis y limitación de la movilidad ocular y del párpado superior izquierdo. Es evaluada por otorrinolaringología destacando compromiso nasosinusal con etmoides y seno frontal izquierdos comprometidos, por lo que se cambia esquema antibiótico a ceftriaxona más clindamicina por 7 días, con diagnóstico de sinusitis complicada. Debido a la ausencia de respuesta favorable es derivada a nuestro centro para evaluación y estudio endoscópico. Laboratorio de ingreso con proteína $\mathrm{C}$ reactiva elevada y leucocitosis discreta. Infectología indica piperaciclina-tazobactam junto con vancomicina y corticoterapia endovenoso.

En el estudio con imágenes destaca: tomografía computada (TC) de órbitas al ingreso en hospital de origen (Figura 1) con compromiso etmoidal, proptosis izquierda y signos sugerentes de celulitis orbitaria y tromboflebitis de la vena oftálmica ipsilateral. Resonancia magnética de cerebro al control en hospital de origen (Figura 2) muestra proptosis izquierda junto a celulitis y compromiso de la vena oftálmica superior ipsilateral.

En cuanto al diagnóstico diferencial; dada la evolución prolongada y respuesta parcial a diversos esquemas antibióticos, hacen poco probable una infección bacteriana clásica, ni una infección fúngica invasiva, por lo que se realiza evaluación multidisciplinaria para completar estudio y manejo. La paciente es evaluada por oftalmología, infectología, reumatología, otorrinolaringología, medicina interna, y neurorradiología, para estudio y manejo. Se maneja patología de base con esquema de insulina NPH e insulina cristalina según esquema. Se realiza estudio extendido con anticuerpos antinucleares (ANA), anticitoplasma de neutrófilos (ANCA), antinucleares extraíbles (ENA) y factor reumatoideo negativos; niveles de complemento C3, C4 y anticuerpo antimieloperoxidasa (MPO) y antiproteinasa 3 (PR3), subclases de inmunoglobulina $G$ 


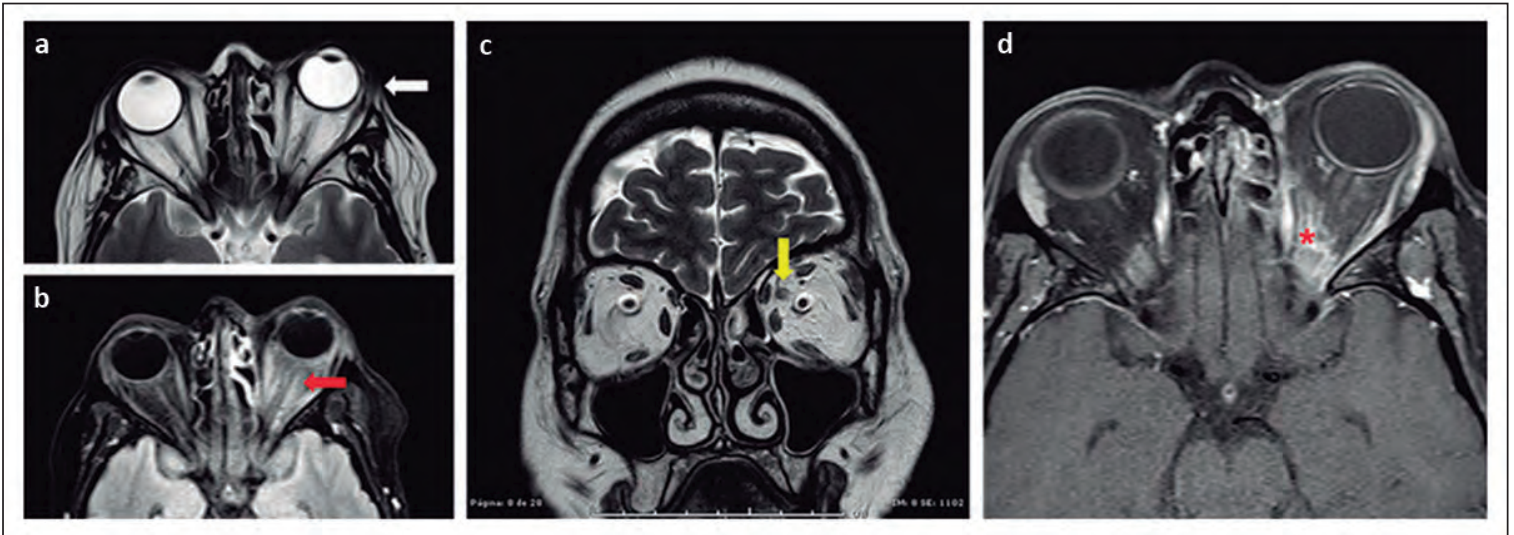

Figura 2. (a) En la resonancia magnética de cerebro, se reconoce proptosis izquierda (flecha blanca). (b) Evaluada la secuencia con inversión de la señal de la grasa y el líquido cefalorraquídeo, se reconoce aumento de señal de grasa intraorbitaria izquierda en relación a celulitis orbitaria izquierda (flecha roja). (c) En la RM de órbitas en secuencia T2 coronal, se demuestra asimetría de calibre de la vena oftálmica superior izquierda en el contexto de tromboflebitis (flecha amarilla). (d) En secuencia T1 FATSAT posterior al uso de gadolinio, se muestra realce intraorbitario intraconal y afectación del vértice orbitario, en el contexto de celulitis orbitaria (asterisco rojo). Moderada proptosis izquierda. Hallazgos concordantes con celulitis orbitaria izquierda y posible tromboflebitis de la vena oftálmica superior.

normales. Además, niveles de vitamina B12, folato, ferritina y saturación de transferrina normales. Serología para virus de hepatitis A, B y C, virus de la inmunodeficiencia humana adquirida y ensayo de inmunospot ligado a enzimas (ELISPOT) para tuberculosis negativos. En el control imagenológico de RM de órbita a los 36 días de su ingreso (Figura 3), persisten hallazgos concordantes con celulitis izquierda y tromboflebitis de la vena oftálmica superior del lado izquierdo.

Se programa biopsia diferida endoscópica por otorrinolaringología en pabellón. Se realiza uncinectomía y antrostomía maxilar izquierda.
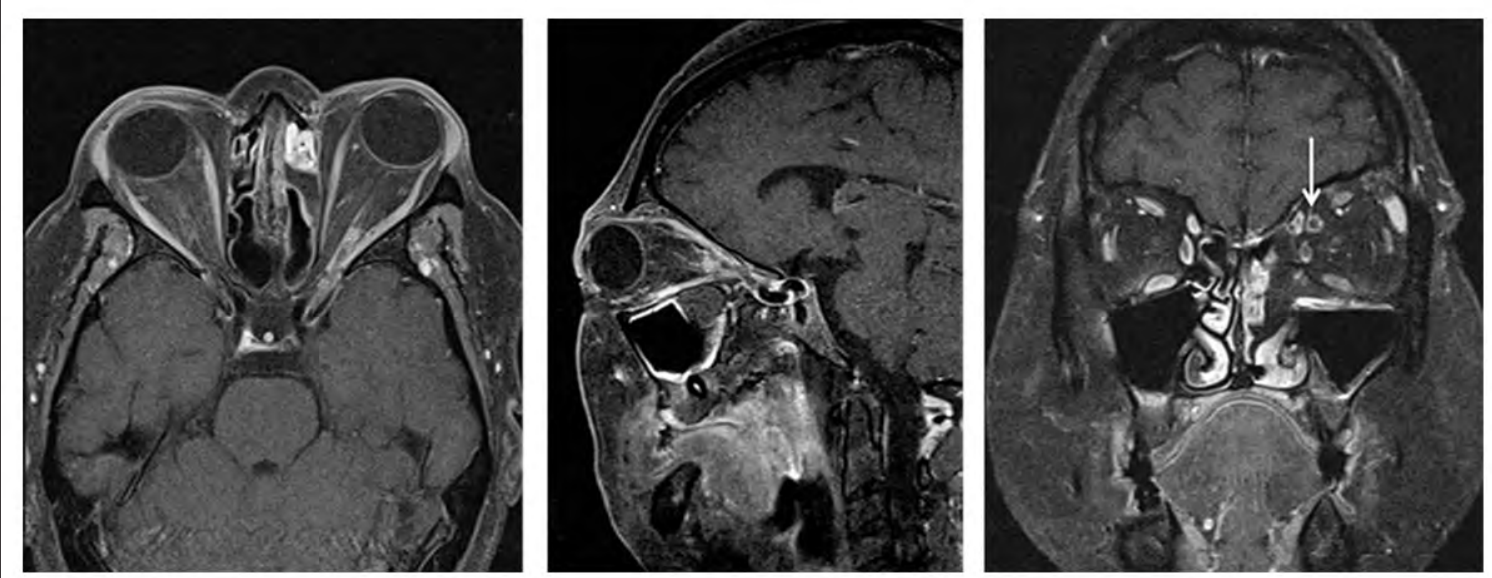

Figura 3. Resonancia magnética de control. En la resonancia magnética de órbitas en secuencia T1 FATSAT posterior al uso de gadolinio, se muestra realce intraorbitario intraconal y afectación del vértice orbitario, en el contexto de celulitis orbitaria, moderada proptosis izquierda. En la imagen coronal destaca dilatación de la vena oftálmica superior con defecto de llene y realce periférico (flecha blanca). Hallazgos concordantes con celulitis orbitaria izquierda y posible tromboflebitis de la vena oftálmica superior. 


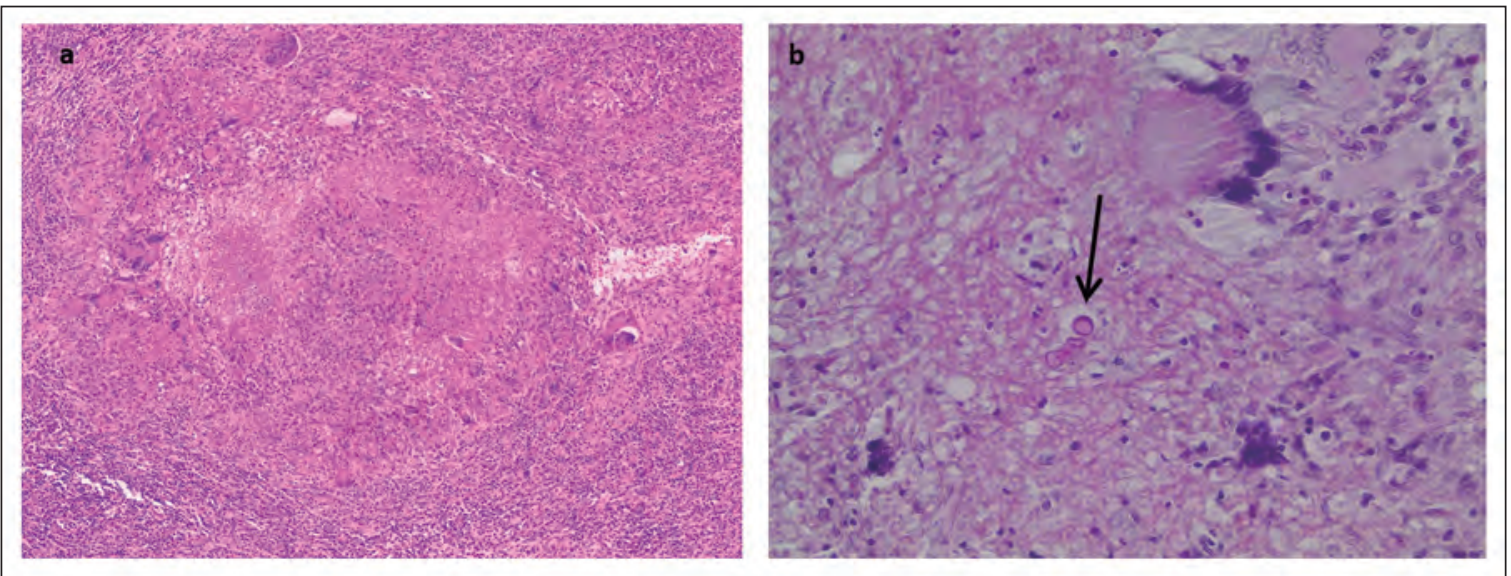

Figura 4. (a) Biopsia diferida. Tinción con hematoxilina eosina $\times 100$. Granuloma con histiocitos, células gigantes, necrosis e inflamación linfocitaria. (b) Tinción con ácido peryódico de Schiff x 400. Necrosis, histiocito, células gigantes, hifa hialina concordante con mucor (flecha negra).

Etmoidectomía anterior izquierda que permite identificar lesión de aspecto polipoideo, de la cual se toma muestra. Se envía a biopsia diferida, PCR universal, PCR para tuberculosis y cultivos corriente de hongos y de Koch. Se identifica dehiscencia de lámina papirácea izquierda que se respeta. Se rescata biopsia diferida que informa mucor sin angioinvasión, presente en trabéculas óseas (Figura 4).

Evoluciona sin complicaciones derivadas de la cirugía. Al examen físico progresa con arreflexia pupilar. Se inicia anfotericina B liposomal $300 \mathrm{mg}$ al día a través de catéter central de instalación periférica. Evoluciona con insuficiencia renal aguda KDIGO 2, acidosis tubular renal distal tipo 1, que se interpreta secundario a tratamiento con antifúngico. Por este motivo, al séptimo día, se disminuye la dosis a $200 \mathrm{mg}(3,2 \mathrm{mg} \mathrm{x} \mathrm{kg})$. Se discute el caso extensamente en forma interdisciplinaria, con paciente y familia cercana, se decide realizar exenteración orbitaria. Once días posterior al inicio de anfotericina, se realiza exenteración orbitaria ampliada, resección amplia de etmoides, lámina papirácea y pared medial del seno maxilar izquierda, sin complicaciones intraquirúrgicas. Es trasladada a unidad de cuidados intensivos, donde evoluciona satisfactoriamente, sin requerimiento de drogas vasoactivas, con buen manejo analgésico y resolución de falla renal, por lo que se aumenta dosis de anfotericina B liposomal a $300 \mathrm{mg}$ al día ( $5 \mathrm{mg} \mathrm{x} \mathrm{kg/día),} \mathrm{que} \mathrm{se} \mathrm{mantiene} \mathrm{hasta}$ alcanzar 6 g. Finalmente, luego de 26 días de tratamiento con terapia endovenosa, se realiza traslape a posaconazol oral $400 \mathrm{mg}$ cada $12 \mathrm{~h}$ por 4 semanas vía oral. El control con TC de órbitas (Figura 5) muestra los cambios postquirúrgicos sin nuevas complicaciones.

Se mantiene en seguimiento y curaciones por equipo de órbita de oftalmología y otorri-

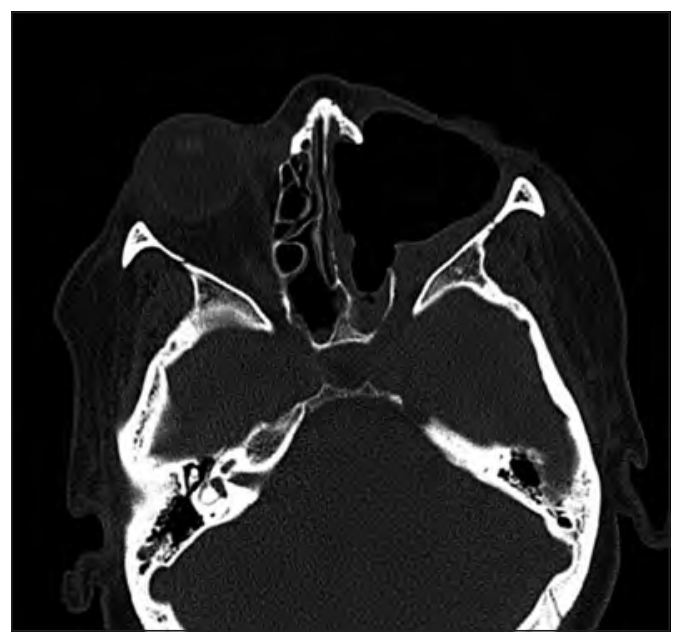

Figura 5. Tomografía computada de órbitas demuestra enucleación izquierda y etmoidectomía, con resección de la lámina papirácea izquierda. 
nolaringología. Luego de 49 días en nuestro centro, la paciente es trasladada a su hospital de origen y es dada de alta a los 2 días a completar posaconazol oral. A los 7 meses de alta, la paciente se encuentra asintomática del punto de vista rinosinusal.

\section{Discusión}

Gutiérrez y cols. realizó una revisión de todos los casos de infección crónica ROC causados por mucorales, publicados en inglés, en Pubmed, desde 1964 hasta 2014. Se identificaron 23 casos. El género predominantemente afectado fue el masculino con 15 casos, la edad promedio de presentación fue 45 años, con un rango de 10 meses a 85 años. El microorganismo no pudo ser aislado en 18 casos. Los casos identificados corresponden a: Rhizopus spp (2), Rhizopus nigrans (1), Mucor irregularis (1), Mucor ramosissimus (1). El tratamiento de elección fue cirugía asociado a anfotericina $B$, con una duración variable de 10 días hasta 8 meses. En el seguimiento, 3 pacientes fallecieron y 3 presentaron recaída entre 11 a 36 meses $^{1}$. Las manifestaciones clínicas iniciales son inespecíficas y variables. Se enumera un listado de síntomas y signos de mucormicosis ROC crónica junto con su porcentaje de presentación en la Tabla 1.

Los síntomas y signos de esta afección pueden ser similares a la forma aguda, sin embargo, destaca la diferencia en el tiempo de presentación ${ }^{9}$. Los síntomas pueden prolongarse por hasta 12 a 24 años previo al diagnóstico ${ }^{1,2,5}$. Resaltamos que si bien, afecta a pacientes con condiciones que generan inmunosupresión, como la diabetes mellitus, también puede afectar a pacientes inmunocompetentes, por lo que el estado inmune no es una herramienta para descartar o no la presencia de una mucormicosis ROC crónica ${ }^{1,2}$. Algunos casos pueden ser asintomáticos, encontrándose como hallazgos en las imágenes, con diagnóstico de mucormicosis no angioinvasiva en la biopsia diferida $^{2}$. La trombosis de la arteria carótida interna y del seno cavernoso son frecuentes en la presentación crónica ${ }^{1,10}$. Otras formas más agresivas incluyen síndrome del ápex orbitario y abscesos cerebrales ${ }^{2,10,11}$. Los pacientes con cetoacidosis diabética, neoplasia hematológica, neutropenia inducida por quimioterapia y uso de deferoxamina, favorecen la angioinvasión fúngica ${ }^{12}$. Los pacientes inmunocompetentes también son susceptibles, pero con formas menos severas ${ }^{2}$. En cuanto al diagnóstico, dentro del laboratorio general, en el hemograma puede haber aumento del recuento de neutrófilos ${ }^{13}$, mientras que los hemocultivos de estos pacientes son negativos ${ }^{14}$.

Es relevante indagar mediante exámenes de laboratorio la presencia de hiperglicemia y cetoasidosis ${ }^{15}$. No existen test serológicos disponibles actualmente para el diagnóstico de mucormicosis ${ }^{7}$.

Los agentes microbiológicos, generalmente identificados, corresponden al género Rhizopus y Mucor. Sin embargo, la identificación del agente se logra sólo en el 11\%-22\% de los casos, lo cual dificulta el diagnóstico ${ }^{1}$. Los mucorales

Tabla 1. Síntomas y signos en mucormicosis rino-órbitocerebral crónica. Adaptado de Prakash y cols., 2019

\begin{tabular}{lc}
\hline Síntomas y signos & $\%$ \\
\hline Fiebre & $26-44$ \\
Cefalea & $5-25$ \\
\hline Edema facial & $27-34$ \\
\hline Algia facial & $3-22$ \\
\hline Descarga nasal & $2-18$ \\
\hline Epistaxis & $0,5-9$ \\
\hline Sinusitis & $1-26$ \\
\hline Hemiplejía & $0-4$ \\
\hline Ulceración nasal & $3-38$ \\
\hline Escara en paladar & $5-14$ \\
Dolor dental & $0,5-3,5$ \\
\hline Entumecimiento facial & $3-7$ \\
\hline Parálisis facial & $0,5-11$ \\
\hline Destrucción ósea y alteración estado mental & $2-22$ \\
\hline Dolor ocular, disminución agudeza visual, & $15-29$ \\
oftalmoplejía & $11-16$ \\
\hline Proptosis & $4,5-9$ \\
\hline Quemosis & $3,5-18$ \\
\hline Ptosis palpebral & $2-16$ \\
\hline Celulitis orbitaria & $2-4$ \\
\hline Necrosis periorbitaria & \\
\hline & \\
\hline
\end{tabular}


se caracterizan por hifas no septadas, con un ángulo de $90^{\circ 1,16}$. El cultivo es difícil de obtener, por lo que se realiza su búsqueda con las tinciones adecuadas en los cortes histopatológicos. Las tinciones útiles para estos fines incluyen: hematoxilina-eosina, ácido peryódico de Schiff y Grocott Gomori ${ }^{1,2,16,17}$. Macroscópicamente el tejido comprometido presenta necrosis ${ }^{2,4}$. Pueden tener formaciones de granuloma, edema de mucosa con infiltración linfocítica, eosinofílica y plasmocitaria ${ }^{1,2,4}$. En el caso presentado, las tinciones de Gram y Grocott revelan presencia de hifas hialinas gruesas no septadas, de contornos irregulares y ángulos de bifurcación diversos, localizados en el centro de los granulomas, compatible con Mucor.

En cuanto al examen físico, los hallazgos en la endoscopía nasal son inespecíficos, con edema del meato medio ${ }^{2}$. La endoscopía nasal puede revelar áreas de necrosis del tabique óseo ${ }^{13}$. Es necesario realizar un adecuado examen oftalmológico, dados los signos que pueden presentar estos pacientes, como se describe en la Tabla 1. En el intraoperatorio puede observarse degeneración polipoidea del cornete medio que, al removerlo, evidencia abundante cantidad de material gris-verdoso similar a la arcilla y necrosis de la mucosa del seno maxilar ${ }^{2,3}$. Estos hallazgos fueron evidenciados en el caso presentado. La ausencia de necrosis en la endoscopía nasal no descarta la mucormicosis crónica ROC.

En el estudio imagenológico, en tomografía computada con contraste, se puede observar erosión del hueso malar con extensión al borde orbitario, cornete medio, arco cigomático y paladar ${ }^{1,3,10,13,16}$. Sin embargo, no es necesaria la presencia de erosión ósea para el diagnóstico, ya que hay casos en los que sólo se observa ocupación heterogénea de las cavidades paranasales, predominantemente, en el seno maxilar en forma unilateral ${ }^{2}$, o masas que refuerzan con contraste en cavidades paranasales, etmoidales o esfenoidales ${ }^{11}$. En el ápex orbitario puede haber ocupación orbitaria por material isodenso a tejidos blandos, que produce proptosis y en las partes blandas; edema preseptal ${ }^{1,10,11}$. La resonancia magnética permite complementar los resultados obtenidos en la TC, siendo de especial utilidad para delinear la extensión intracraneana, que puede incluir: trombosis del seno cavernoso, masa intracraneana, absceso cerebral, zonas de isquemia ${ }^{11}$. El seguimiento con imágenes es fundamental, especialmente en pacientes con compromiso orbitario ${ }^{2}$. El diagnóstico diferencial debe incluir mucormicosis invasiva, neoplasia, sinusitis bacteriana, trastornos granulomatosos, trombosis del seno cavernoso, pseudotumor.

El tratamiento del mucor crónico implica una serie de intervenciones que van desde la corrección de factores predisponentes como la hiperglicemia y/o inmunosupresión hasta el uso de antifúngicos y cirugía amplia de debridamiento, en que muchas veces hay que recurrir a la exenteración orbitaria. Como se menciona en la introducción, la literatura se basa en series de casos, por lo que el tratamiento no está estandarizado. Algunos autores plantean el uso de anfotericina B o cirugía como monoterapia o en forma complementaria ${ }^{1,2,13}$ por lo que, el tratamiento debe decidirse en forma multidisciplinaria analizando cada caso en particular. Los niveles elevados de glucosa en sangre contribuyen a la patogenia de infección por mucor ${ }^{12}$, por lo que su manejo es fundamental en el tratamiento. Debido al bajo número de pacientes, no existe un protocolo que compare diferentes enfrentamientos de manejo de glicemia específicamente en este grupo. El equipo de nutrición y diabetes indicó en nuestro paciente insulina NPH y cristalina. La anfotericina B es el medicamento usado con mayor frecuencia. En su forma liposomal es preferible por su menor toxicidad y mejor tolerancia, así como mayor eficacia en comparación a otras formulaciones de anfotericina B. También se reporta el uso de ketoconazol, posaconazol, isavuconazol y griseofulvina. La dosis y duración de la anfotericina $B$ es variable en la literatura. Para la formulación no liposomal, la dosis habitual es $1 \mathrm{mg} / \mathrm{kg} /$ día hasta una dosis acumulada de 2,5 a $3 \mathrm{~g}$, mientras que para la formulación liposomal se recomienda una dosis de $5 \mathrm{mg} / \mathrm{kg} /$ día endovenosa hasta conseguir la resolución de la enfermedad, aunque se han llegado a utilizar dosis de 7,5 a $10 \mathrm{mg} / \mathrm{kg} /$ día ${ }^{1,2,10,14,15}$. Frente a una respuesta satisfactoria al tratamiento endovenoso, puede completarse con posaconazol o isavuconazol oral hasta un plazo incluso mayor a 6 meses $^{14}$. También está descrito en la literatura el uso de antifúngicos locales ${ }^{10,15}$. Los abordajes quirúrgicos utilizados para el manejo de la afectación rinosinusal 


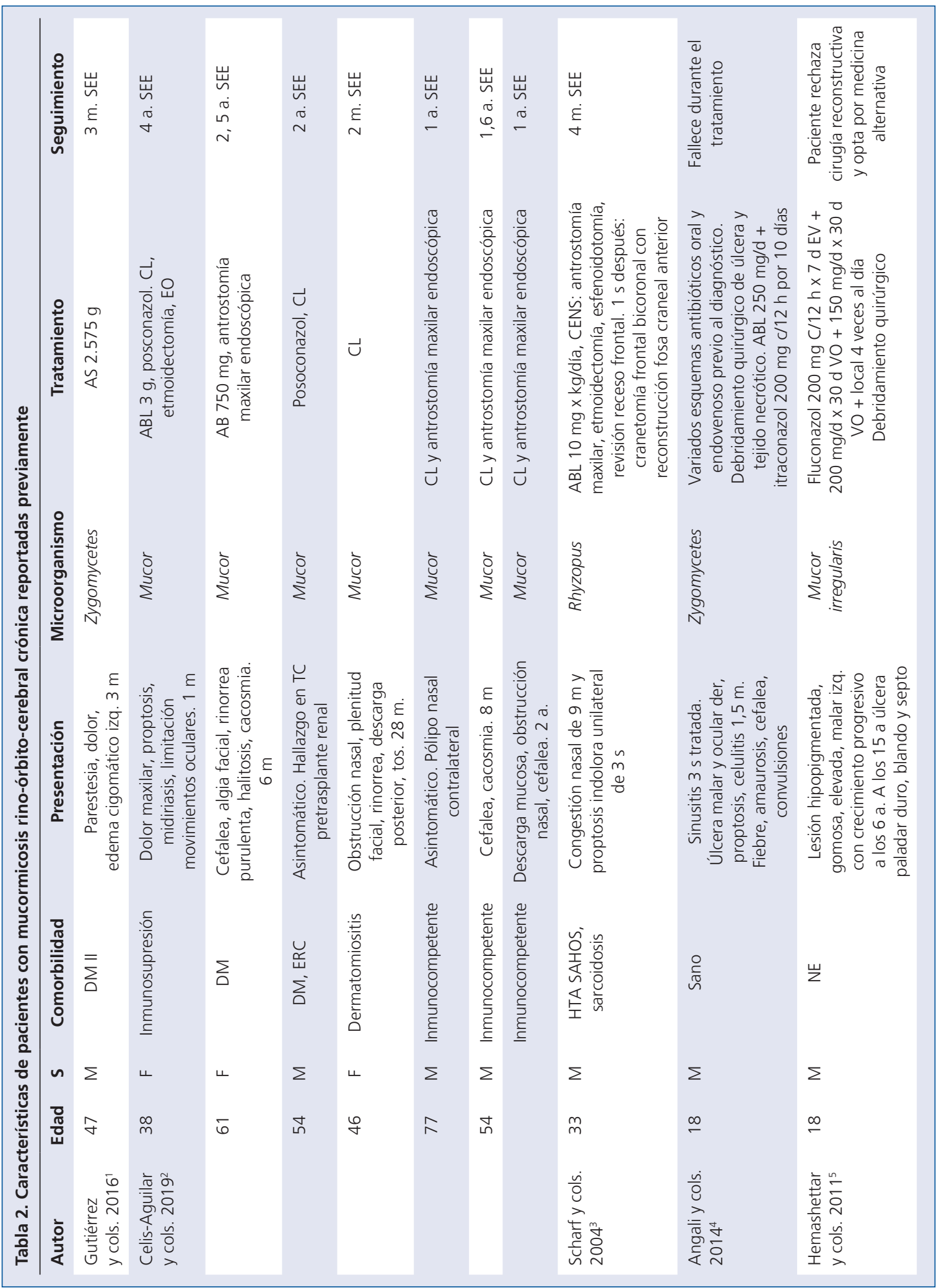




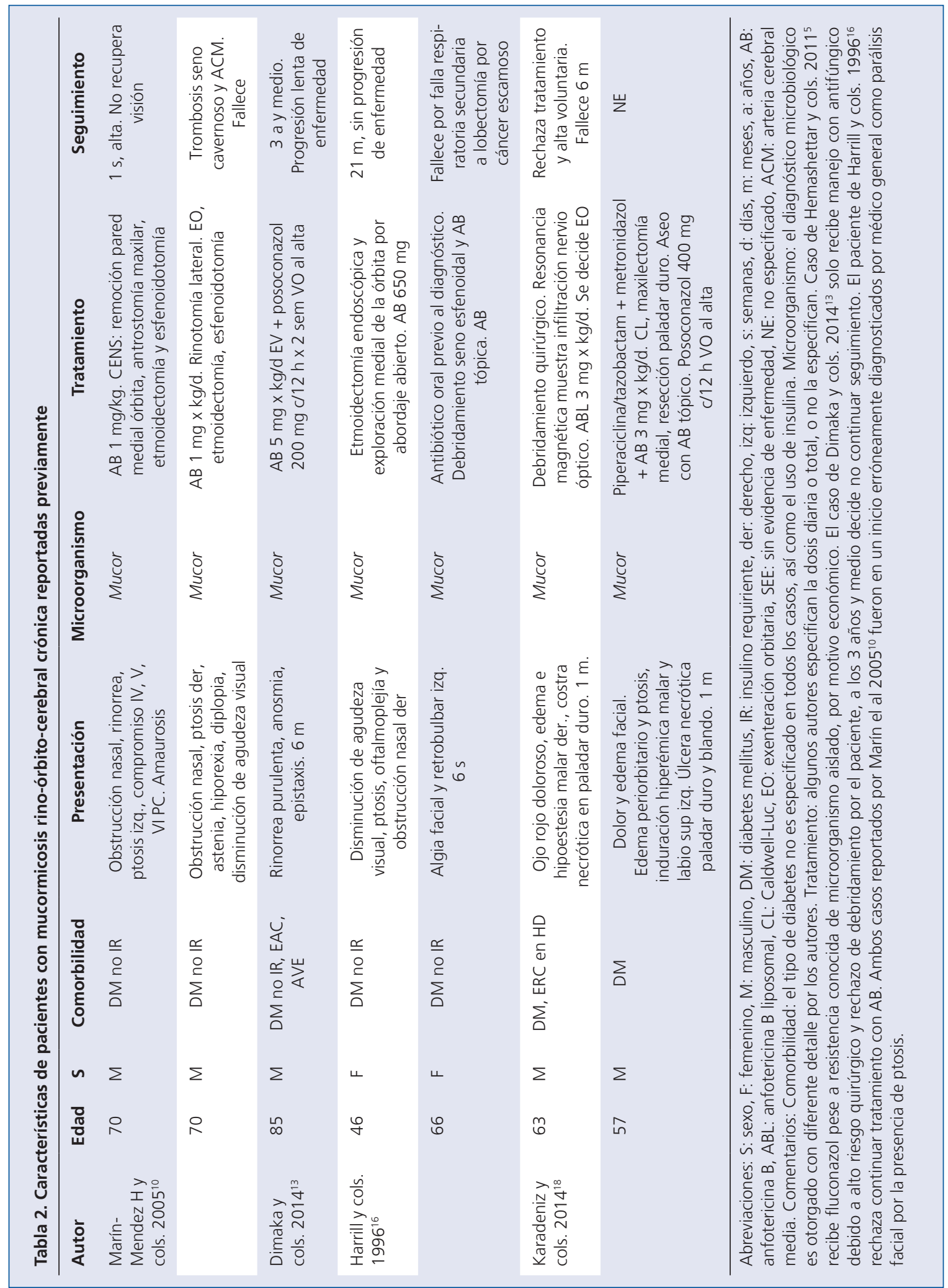


son variables, dependiendo de la ubicación y extensión de la lesión. En caso de compromiso maxilar, se menciona reiteradamente el uso de Caldwell-Luc L $^{1,2,10,16,18}$. Aquellos casos con compromiso intracraneano pueden requerir abordajes abiertos extensos y reparación de la base de cráneo ${ }^{3}$. Por lo que los neurocirujanos deben estar al tanto de estos pacientes según corresponda. El caso presentado fue manejado exitosamente con abordaje extenso endoscópico. Esto requiere adecuado instrumental quirúrgico y entrenamiento en cirugía endoscópica nasosinusal dada la complejidad que significan estos casos, debido a la alteración anatómica secundaria al compromiso fúngico crónico.

La exenteración orbitaria es una cirugía mutilante para el paciente, con secuelas estéticas, funcionales y psicológicas. Pese a esto, en aquellos casos con compromiso orbitario progresivo y confirmación de patología intraconal, los autores lo recomiendan dado la alta mortalidad agregada ${ }^{2,10,18}$. En una serie chilena publicada en esta revista en 2008, que incluyó 16 pacientes con mucormicosis ROC, dos de ellos en forma crónica, en seis pacientes se realizó exenteración orbitaria, uno de los cuales fue de presentación crónica, todos con compromiso de la agudeza visual severa9. El compromiso intraconal incluye la presencia de masas focales intraorbitarias y necrosis generalizada. Tanto los hallazgos clínicos como imagenológicos son necesarios para la toma de decisión ${ }^{10,18}$. Por otra parte, la exenteración orbitaria en pacientes con esta indicación puede empeorar la sobrevida, probablemente asociado a la agresividad de la patología más que la cirugía en sí misma ${ }^{15}$. El manejo conservador es preferible frente a compromiso muscular extraocular aislado, con visión preservada y en ausencia de progresión ${ }^{19}$. Concordamos con los autores que utilizan la cirugía con debridamiento quirúrgico amplio rinosinusal, que en este caso requirió, además, exenteración orbitaria, junto con anfotericina $\mathrm{B}$ endovenoso y continuación con antifúngico oral al alta ${ }^{1-3}$ con buen resultado en el caso presentado.

La recaída se define como nueva sintomatología en 4 semanas o menos posterior a la resolución de los síntomas, por otra parte, recurrencia si es luego de la quinta semana.
Éstas se presentan en el $13 \%$ y $33 \%$ de los casos respectivamente ${ }^{1}$. La recurrencia se presenta en forma tardía, hasta 3 años después del diagnóstico, por lo que resulta fundamental el seguimiento ${ }^{1}$. No está establecido cuál es el mejor método de seguimiento, pero resulta fundamental controlar al paciente por un período prolongado. En la Tabla 2 se resumen los datos epidemiológicos y esquemas de tratamiento, que están disponibles, en base a los casos publicados por los autores incluidos en esta discusión, para que el lector pueda realizar un análisis detallado y eventualmente servir como referencia al enfrentarse a un caso similar.

\section{Conclusiones}

La mucormicosis ROC crónica es una patología poco frecuente, y dada su clínica inicial poco específica, puede ser subdiagnosticada, con lo cual, el diagnóstico puede tardar meses, incluso años. Se debe tener un alto índice de sospecha, no solo en pacientes inmunocomprometidos, ya que, como expusimos, puede presentarse en pacientes inmunocompetentes. El diagnóstico resulta complejo y debe considerarse la clínica, examen físico otorrionolaringológico y oftalmológico completo, junto con imágenes. Debido a la morbimortalidad asociada es necesario evaluación y manejo multidisciplinario, en el cual, nuestra especialidad juega un rol fundamental.

\section{Bibliografía}

1. Gutiérrez-Delgado EM, Treviño-González JL, Montemayor-Alatorre A, et al. Chronic rhinoorbito-cerebral mucormycosis: A case report and review of the literature. Ann Med Surg (Lond) 2016;6:87-91. Disponible en https://doi.org/10.1016/j. amsu.2016.02.003.

2. Celis-Aguilar E, Burgos-Páez A, Villanueva-Ramos N, et al. An Emergent Entity: Indolent Mucormycosis of the Paranasal Sinuses. A Multicenter Study. Int Arch Otorhinolaryngol 2019;23:92-100. Disponible en https://doi.org/10.1055/s-0038-1667005

3. Scharf JL, Soliman AM. Chronic rhizopus invasive fungal rhinosinusitis in an immunocompetent host. Laryngoscope 2004; 114:1533-1535. doi: 10.1097/00005537-200409000-00005. 


\section{CASO CLÍNICO}

4. Angali RK, Jeshtadi A, Namala VA, Gannepalli A. Fatal rhino-orbito-cerebral mucormycosis in a healthy individual. J Oral Maxillofac Pathol 2014; 18:460-463. Disponible en https://doi. org/10.4103/0973-029X.151355.

5. Hemashettar BM, Patil RN, O’Donnell K, Chaturvedi V, Ren P, Padhye AA. Chronic rhinofacial mucormycosis caused by Mucor irregularis (Rhizomucor variabilis) in India. J Clin Microbiol 2011; 49:2372-2375. Disponible en https://doi. org/10.1128/JCM.02326-10.

6. Prakash H, Chakrabarti A. Global Epidemiology of Mucormycosis. J Fungi (Basel) 2019;21:5. doi: 10.3390/jof5010026.

7. Richardson M, Page I. Role of Serological Tests in the Diagnosis of Mold Infections. Curr Fungal Infect Rep 2018; 12:127-136. Disponible en https://doi. org/10.1007/s12281-018-0321-1.

8. Álvarez Duarte E, Denning DW. Serious fungal infections in Chile. Eur J Clin Microbiol Infect Dis 2017; 36:983-986. Disponible en https://doi. org/10.1007/s10096-017-2925-8.

9. Rahal M, Moreno M, Villa J. Mucormicosis rino-orbito-cerebral: experiencia en 12 años. Rev Otorrinolaringol Cir Cabeza Cuello 2008; 68:27-34.

10. Marín-Méndez H, Monroy-Aguirre D, RodríguezPerales M, Caretta Barradas S. Síndrome de ápex orbitario causado por mucormicosis órbito-cerebral crónica e indolente: reporte de dos casos. An ORL Méx 2005; 50:64-68.

11. Mohindra S, Mohindra S, Gupta R, Bakshi J, Gupta SK. Rhinocerebral mucormycosis: the disease spectrum in 27 patients. Mycoses 2007; 50:290-296. Disponible en https://doi.org/10.1111/j.1439- 0507.2007.01364.x.

12. Ibrahim AS, Kontoyiannis DP. Update on mucormycosis pathogenesis. Curr Opin Infect Dis 2013; 26:508-515. 10.1097/QCO.0000000000000008.

13. Dimaka K, Mallis A, Naxakis SS, et al. Chronic rhinocerebral mucormycosis: a rare case report and review of the literature. Mycoses 2014; 57:699-702.

14. Ruiz Camps I, Salavert Lletí M. El tratamiento de la mucormicosis (cigomicosis) en el siglo XXI. Rev Iberoam Micol 2018; 35:217-221.

15. Nithyanandam S, Jacob MS, Battu RR, Thomas RK, Correa MA, D'Souza O. Rhino-orbitocerebral mucormycosis. A retrospective analysis of clinical features and treatment outcomes. Indian J Ophthalmol 2003; 51:231-236.

16. Harril WC, Stewart MG, Lee AG, Cernoch P. Chronic rhinocerebral mucormycosis. Laryngoscope 1996; 106:1292-1297. Disponible en https://doi. org/10.1097/00005537-199610000-00024.

17. Sachdeva K. Rhino-oculo Cerebral Mucormycosis with Multiple Cranial Nerve Palsy in Diabetic Patient: Review of Six Cases. Indian J Otolaryngol Head Neck Surg 2013; 65:375-379. Disponible en https://doi. org/10.1007/s12070-013-0659-1.

18. Karadeniz Uğurlu Ş, Selim S, Kopar A, Songu M. Rhino-orbital Mucormycosis: Clinical Findings and Treatment Outcomes of Four Cases. Turk J Ophthalmol 2015; 45:169-174. Disponible en https:// doi.org/10.4274/tjo.82474.

19. Peterson K, Wang M, Canalis R, Abemayor E. Rhinocerebral Mucormycosis: Evolution of the Disease and Treatment Options. Laryngoscope 1997; 107:855-862. Disponible en https://doi. org/10.1097/00005537-199707000-00004. 